\title{
From Contextual Inquiry to Designable Futures: What Do We Need to Get There?
}

\author{
Sidney W.A. Dekker, Linköping Institute of Technology \\ James M. Nyce, Emporia State University \\ Robert R. Hoffman, Institute for Human and Machine Cognition
}

\begin{abstract}
$\infty$ o, what is an end user, really? And what do we really mean by the procurement process? Human factors
\end{abstract} researchers often take certain agendas, terms, and theories for granted or rely on them out of habit. This essay takes

a special look at contextual enquiry as a putatively (and indeed potentially) superior way of giving end users a serious say in the procurement process of complex cognitive systems.

\section{"End user" \\ Human-centered systems result when software engi- neers or developers give attention to the orientations, expec- tations, and understandings of the people who will be part of the sociotechnical system. ${ }^{1}$ But this is far easier said than done. What are the orientations, expectations, and understandings of the "end user"? The term presupposes that humans and machines can and should be treated sepa- rately_assessed by different criteria. "End user" is a hang- over label from computer science of the 1980s; it contra- dicts the systems stance of human-centered computing and the Triples rule, which specifies that stance. ${ }^{2}$ Also, the term suggests that users involved in the design team are those, and only those, who will actually operate the machines. This is not necessarily true.}
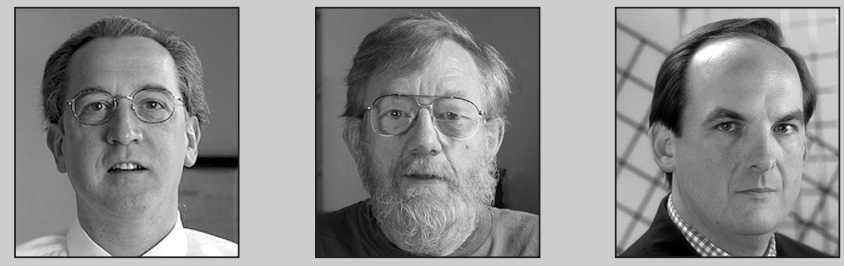

Editors: Robert R. Hoffman, Patrick J. Hayes, and Kenneth M. Ford Institute for Human and Machine Cognition, University of West Florida rhoffman@ai.uwf.edu
Take weather forecasting, for instance. To design a new workstation system to aid forecasters, the designers might rely on forecasters' participation in

- Identifying leverage points and decision requirements (the cognitive work analysis phase)

- Identifying and refining potential design innovations

- Evaluating and reprototyping

But if the new system does not help forecasters in their own work (for instance, evaluating forecast accuracy) and in the work of creating products that help their customers (pilots or the general public, for example), its usefulness will be restricted. Where is the "end" in "end user"?

\section{"Procurement process"}

Merely involving end users in the design process is not a panacea. Neither is overreliance on verification and validation, or on "getting human factors included early." Designs are hypotheses about an "envisioned world." The Envisioned World Principle that David Woods and Sidney Dekker proposed can be stated as

\footnotetext{
The introduction of new technology, including appropriately human-centered technology, will bring about changes in environmental constraints (that is, features of the sociotechnical system, or the context of practice). Even though the domain constraints may remain unchanged, and even if cognitive constraints are leveraged and amplified, changes to the environmental constraints may be negative.
}

Testing a "design as hypothesis" ultimately requires fielding the system, but this means prototyping the system at various levels (specific interfaces, software, user training, and so on). By this time, so much commitment and cost (psychological, organizational, political, financial) are involved that the potential for changing the design on the basis of end user feedback becomes almost impossible. Making things even more complex is what we call the Moving Target Rule: 
The sociotechnical workplace is constantly changing, and constant change in environmental constraints may require constant adaptation in cognitive work, even if domain constraints remain constant.

In other words, by the time you are ready to test an envisioned-world hypothesis, the sociotechnical workplace in which the work will be carried out will already have changed. Frustrations with the procurement process have led human factors researchers to frontload by trying to achieve a rich understanding of the nature of practice before beginning to develop new technology.

Front-loading, however, might not be able to address the dilemma that the Moving Target Rule poses for designers and developers. With the promise of privileged access to an understanding of what activities mean to the people who do them, designers have sought enlightenment by one or another form of contextual inquiry. ${ }^{4}$ This includes bringing to bear methods from ethnography ${ }^{1,5}$ and cognitive work analysis. These approaches involve finding out about people's work, about where they are doing that work while they are doing it, and about what doing that work means to them. Design, by extension, is not so much about building artifacts or systems as about creating new ways to work.

\section{Is contextual inquiry really enough?}

Ethnographers have often have failed to provide meaningful input into designers' choices. ${ }^{6}$ They typically have difficulty jumping from the description and analysis of current practice to requirements capture, in part because of how ethnography has been defined as a method, but also because of analytic choices ethnographers have made with respect to design problems and design issues.

Those involved in design and development (including ethnographers) are often tempted to equate what informants do or tell them with what ethnography is and can tell them. Confounding informant understanding with ethnographic analysis has profound implications for ethnography's credibility and the contribution it can make to the creation of human-centered systems.

Of course, informants can claim privileged access to their operating world: What end users know and can tell us always has to be right in a sense; otherwise, they could not carry out their work. However, no set of practitioners, however expert, can be expected to be able to easily articulate analytic frameworks or categories strong enough to specify a complete design agenda. This is not to deny practitioners insight or analytic ability; it just acknowledges that experts in modern organizations are laypeople most of the time, no matter how reflective they are. According to Helena Karasti, "practitioners take the most fundamental aspects of their ordinary work practices for granted. It is not their task but the fieldworker's to reveal and make visible aspects of work practice that practitioners cannot make explicit." ${ }^{, 7}$ In short, the analysis of complex work requires considerable second-order analysis to yield data and insight relevant to a given problem and design agenda.

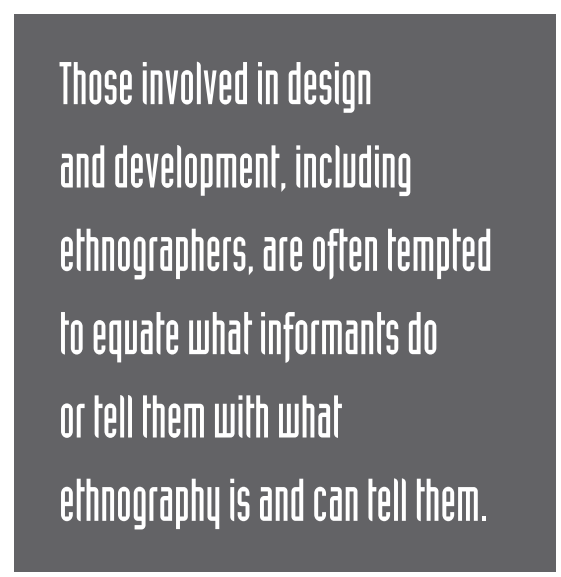

Tying ethnography to pragmatic design hinges on two things:

- Understanding work's features and objects, not just cast in the informant's language but also recast by extracting, revising, and verifying the categories by which informants make sense of their world

- Finding ways to "build out" this revised, nuanced understanding into designable technology

Strong ethnography is more than "record and replay." The ethnographer must move back and forth between informant understandings ("native categories" is the term used in ethnography) on the one hand and analyses of the native understandings and categories on the other hand. This kind of ethnography must be informed by what designers require when they talk and think about human-centered requirements regarding work and systems. "To build out to the future"-in other words, to address the Moving Target Rule-requires that we have a principled understanding of the work that informants do and the resources they use to achieve it. It is only from knowledge such as this that we can extrapolate into the future.

Let's look at two contextual inquiries of a paper-based artifact that many system developers have wanted to replace with computerized variants: the air traffic control flight progress strip, which air traffic controllers use to display important flight data for a particular flight (planned altitude, destination, route, and so on). We examine these inquiries especially for their ability to go from contextual finding in the present to a designable future.

\section{Study I: Strips help controllers build their mental picture of air traffic}

Hailed as the exemplar of contextual flight progress strip studies, ${ }^{5}$ a Lancaster University project ${ }^{6}$ spent many person-months on the ethnographic observation and analysis of air traffic controller practice, with a focus on flight strip use. The grand conclusion of this often-cited project was that strips

\section{are the means by which controllers see and note what is happening, what they have already done, what needs to be done. They are an essential feature of "getting the picture," "organizing the traffic," which is the means of achieving the orderliness of traffic. The strips and their organization are a proxy orderliness of the configuration of the traffic flow. ${ }^{6}$}

Strips help controllers "get the picture." Fine. But such an insight should not have taken longer to arrive at than half an afternoon spent observing air traffic controllers. This raises the question about what kind of ethnography the researchers were practicing. When we reduce ethnography to (or take it as) mere observation and description, we ignore the need to move to second-order work. In other words, neither the categories nor the "positions" of the natives (the people being studied) or the investigators themselves become objects of any sustained inquiry. The result is that this kind of ethnography never moves much further than the reproduction of what, for the investigators and informants, is common sense. Furthermore, if strips are adequate for letting a controller know what is going on, then there is no point in automating or developing anything new.

Such ethnography then, intentionally or not, privileges the status quo, even valorizing standard practice. It should be no won- 
der that designers often think they can do just as well or better themselves. As if to confirm the point, when the air traffic control system developers lost patience with the ethnographers, they gave them a set of guiding questions to answer, so that they (the developers) could get on with building their electronic substitute: ${ }^{6}$

- What characteristics of the existing manual system are unimportant and need not be supported in a computerized system?

- What are important manual activities that need not be supported in a computerized system because the activities are a consequence of the fact that no computer support is available?

- What characteristics of the manual system must be replicated without change in a computerized system?

- What activities from the manual system may be supported in a way which is different from that used in the manual system?

The developers missed the point. All the old biases of optimistic engineered positivism are here to see. The developers believed that they could simply substitute computers for paper-they just needed the ethnographers to tell them which parts to swap. The failure of ethnographers and developers to get along, or even understand each other, is not unique to the Lancaster project. ${ }^{1}$ Confronted with challenges about such machine-oriented silliness, the ethnographers never quite recovered. Nor could they, given the way ethnography was defined in this project-as not requiring any second-order, analytic work.

The Lancaster project presents a particularly naïve form of ethnography: The researchers did not interrogate what was common sense to the air traffic controllers (or to themselves). They took practitioner categories as canonical and inherently correct, not requiring any second-order analysis. Informant competence, as expressed in domain terms and categories, can be strong and valid. It can also represent misconceptions or apocrypha.

Mistaking the domain practitioner's statements and categories for contextual understanding or analysis does not lead to strong ethnography. Nor does it generate meaningful design guidance. Tellingly, the title of the Lancaster project paper is "From Ethnographic Record to System Design ...," not "From Ethnographic Analysis ...." Clearly, ethnographic recording is not enough.
Unless ethnography takes on the analytic question "What is really going on here?" the jump from ethnographic record to system design is too great for designers to deal with. Designers cannot and do not want to deal with it-indeed, it should not be their job.

On the other extreme, in their book on contextual design, ${ }^{4}$ Hugh Beyer and Karen Holtzblatt prohibit the use of domain categories in design discussions, lest such characterizations trap developers into assumptions or lure them into believing that practitioner statements (such as "flight strips help me get the mental picture") can actually serve as design principles or analytic statements. Talking to designers meaningfully requires the person conducting the contextual inquiry

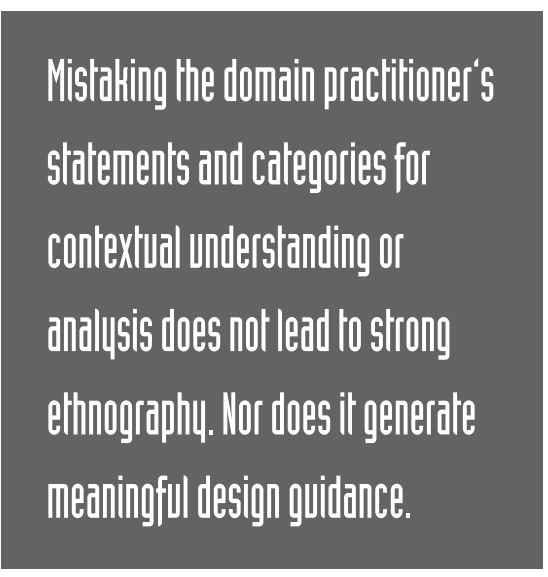

to engage in a kind of strong ethnography, strong particularly in its analysis. ${ }^{1}$ Only such higher-order analytical work can lead to designable futures. The question is, how?

\section{Study II: Strips help compress complexity, manage dynamics, and support coordination}

Informant remarks such as "flight strips

help me get the mental picture" should serve as the starting point of a contextual inquiry, not as its conclusion. The revision of categories is a hallmark of strong ethnography, and Geoff Ross's study of flight progress strips in Australia can serve as an example. ${ }^{8}$ Ross conducted a survey that many ethnographers derided for imposing the researcher's meaning on data rather than bringing out the domain practitioner's. But Ross conducted a stronger analysis and synthesis. He slowly treaded through the masses of survey data, abstracting and categorizing as he went along, relying at each step on previous categorizations for help. ${ }^{9}$
Ross's study shows the strength of second-order analytic work. It involved multiple intermediate steps of analysis and synthesis, each with an explicit trace that others could follow and critique. The typical way proposed is to move up from contextspecific details to concept-dependent generalizations in successive steps-each more abstract than the previous one, each stated less in domain terms and more in design terms. ${ }^{4,10}$ Furthermore, both the domain and design terms that emerge are grounded in the literature (that is, in prior analytic and design experience) as much as they are in informant meaning and understandings. This stepwise movement from context to concept is crucial for moving to designable futures, for linking ethnographic analysis and robust design guidance.

To use an example from Ross's study, context-specific controller activities such as "entering a pilot report; composing a flight plan amendment" reveal an intentional strategy (a slightly higher level of analysis), which is the "transformation or translation of information for entry into the system." "At an even higher level of analysis, this could be referred to as "coding" activity. Part of this coding is symbolic, in that it uses highly condensed markings on flight strips (red underlinings, black circles) to denote and represent "what is going on." Only from there can we make the (then no longer so large) jump to the highest level of abstraction-helping us identify the flight strip's role in making sense of workplace and task complexity. Unable to keep straight all the details of what a flight might do, the controller compresses complexity by letting one symbol stand for complex concepts and interrelationships, some even temporal.

Other high-level, concept-dependent roles of the flight strip would be to anticipate dynamics (what comes next) and support coordination (for example, in handing over flights to other controllers). Note how the language is no longer cast in that of context-specific details or artifact-related practices. Instead, second-order analysis provides developers with more general or abstract concepts that are informed by what these workers find to be significant in their work and world. It is with these concepts and categories that developers can open a window to a designable future.

Complexity and dynamics, as well as coordination, are critical features that make 
air traffic control what it is, including difficult. Developers must take into account the fact that controllers use their artifacts to help them deal with complexity, anticipate dynamic futures, and coordinate with other controllers. Ross shows us the paradox in contextual inquiry for HCC: Creating designable futures requires sensitivity to context. Yet it asks us to extract the description of people's work away from the current context that shapes it. Otherwise, designers will not understand what the ethnographic analyst is trying to say, so they will not know what to do next. This contrasts with the Lancaster project, which mistook the domain practitioners' language, categories, and understanding for analytic substance, and thereby assumed that the practitioners were self-reflective enough to do their ethnographic work for them. Strong ethnography is both analysis and synthesis, a working back and forth between informant statements and categories and concepts that capture reality and meaning.

D esignable futures, and by extension HCC systems, can result if we succeed in describing people's work in terms that let designers proactively understand, even anticipate, the challenges of that work. Designers do not build artifacts or systems so much as they create new ways in which practitioners must handle the challenges associated with work. Involving users in the design and procurement process does not guarantee meaningful design input. Contextual inquiry, as a popular way of involving users, must not mistake informant understanding for analytic senses of work. Like any human performance data gathering, it must be backed up by strong, second-order analysis, lest designers and developers get misguided.

\section{References}

1. R.J. Anderson, "Representations and Requirements: The Value of Ethnography in System Design," Human-Computer Interaction, vol. 9, no. 2, 1994, pp. 151-182.

2. R.R. Hoffman et al., "The Triples Rule," IEEE Intelligent Systems, vol. 17, no. 3, May/June 2002, pp. 62-65.

3. D.D. Woods and S.W.A. Dekker, "Anticipating the Effects of Technology Change: A New Era of Dynamics for Human Factors," Theo- retical Issues in Ergonomics Science, vol. 1, no. 3, 2001, pp. 272-282.

4. H. Beyer and K. Holtzblatt, Contextual Design: Defining Customer-Centered Systems, Academic Press, 1998.

5. R.H.R. Harper, "The Organisation in Ethnography: A Discussion of Ethnographic Fieldwork Programs in CSCW," Computer Supported Cooperative Work, vol. 9, no. 2, May 2000, pp. 239-264.

6. J.A. Hughes, D. Randall, and D. Shapiro, "From Ethnographic Record to System Design: Some Experiences from the Field," Computer Supported Cooperative Work, vol. 1, no. 3, 1993, pp. 123-141.

7. H. Karasti, Increasing Sensitivity towards Everyday Work Practice in System Design, doctoral dissertation no. A 362, Dept. Information Processing, Univ. Oulu, 2001, p. 66.

8. G. Ross, Flight Strip Survey Report, The Australian Advanced Air Traffic System Operations Instructor, Air Traffic Services Australia, 1995.

9. P.S. Della Rocco, C.A. Manning, and H. Wing, Selection of Air Traffic Controllers for Automated Systems: Applications from Current Research, tech. report DOT/FAA/AM-90/13, Nat'l Technical Information Service, Springfield, Va., 1990.

10. Y. Xiao and K.J. Vincente, "A Framework for Epistemological Analysis in Empirical (Laboratory and Field) Studies," Human Factors, vol. 42, no. 1, 2000, pp. 87-101.

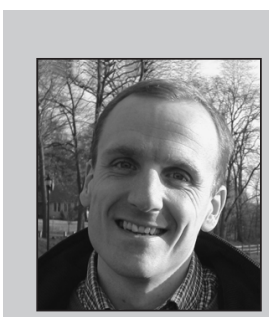

Sidney W.A. Dekker is an associate professor at the Linköping Institute of Technology and director of studies at the Center for Human Factors in Aviation. Contact him at Linköping Inst. of Technology, SE-581 83, Linköping, Sweden; sidde@ikp.liu.se.

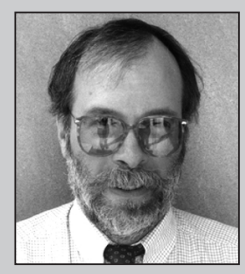

James M. Nyce is an associate professor in the School of Library and Information Management at Emporia State University, an adjunct associate professor in the Department of Radiology at the Indiana University School of Medicine, and a docent at Linköping University. Contact him at $9219 \mathrm{~W} 1250 \mathrm{~N}$, Albany, IN 47320; jnyce@ rocketmail.com.

Robert R. Hoffman is a research scientist at the University of West Florida's Institute for Human and Machine Cognition and a faculty associate in the Department of Psychology. Contact him at the IHMC, 40 Alcaniz St., Pensacola, FL 32501; rhoffman@ai.uwf.edu.

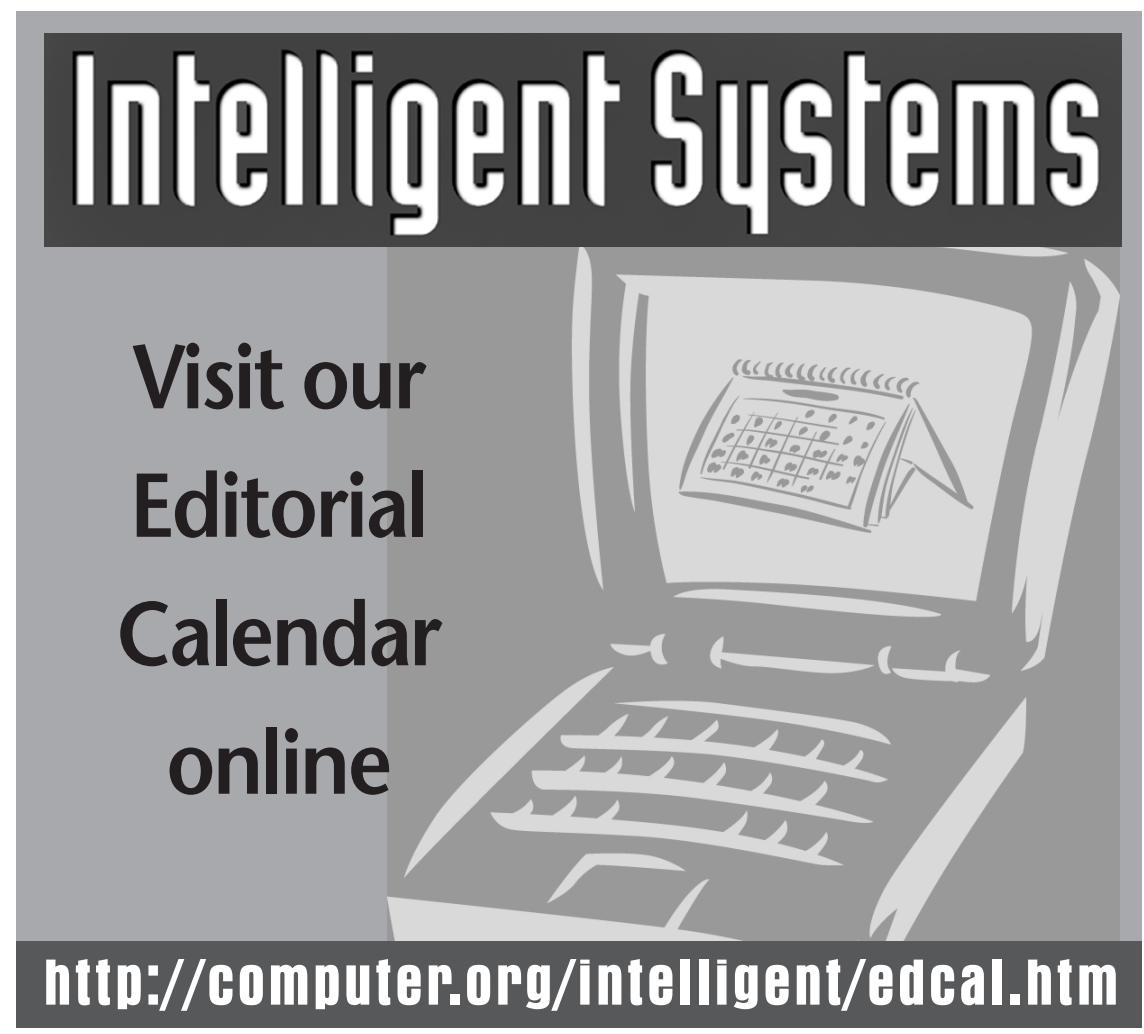

